\title{
EXPLOITING AUTO-COLLIMATION FOR REAL-TIME ONBOARD MONITORING OF SPACE OPTICAL CAMERA GEOMETRIC PARAMETERS
}

\author{
Wei Liu ${ }^{1,}$ *, Hui Wang ${ }^{2}$, Dan Liu ${ }^{2}$, Yuzhe Miu ${ }^{1}$ \\ ${ }^{1}$ Xi'an Research Institute of Surveying and Mapping, No.1 Middle Yanta Road, Xi'an, China, 710054-1271772072@qq.com \\ ${ }^{2}$ State Key Laboratory of Integrated Service Network, Xidian University, Xi'an, China, 710071-huiwang_ii@yeah.net \\ ${ }^{2}$ State Key Laboratory of Integrated Service Network, Xidian University, Xi'an, China, 710071-ada6963@163.com \\ ${ }^{1}$ Xi'an Research Institute of Surveying and Mapping, No.1 Middle Yanta Road, Xi'an, China, 710054-442937352@qq.com
}

\section{Commission II, WG II/1}

KEY WORDS: Optical Auto-collimation, Geometric Parameters, Onboard Monitoring, On-orbit Calibrate, Space Optical Camera, Autocollimator

\begin{abstract}
:
Precise geometric parameters are essential to ensure the positioning accuracy for space optical cameras. However, state-of-the-art onorbit calibration method inevitably suffers from long update cycle and poor timeliness performance. To this end, in this paper we exploit the optical auto-collimation principle and propose a real-time onboard calibration scheme for monitoring key geometric parameters. Specifically, in the proposed scheme, auto-collimation devices are first designed by installing collimated light sources, area-array CCDs, and prisms inside the satellite payload system. Through utilizing those devices, the changes in the geometric parameters are elegantly converted into changes in the spot image positions. The variation of geometric parameters can be derived via extracting and processing the spot images. An experimental platform is then set up to verify the feasibility and analyze the precision index of the proposed scheme. The experiment results demonstrate that it is feasible to apply the optical auto-collimation principle for real-time onboard monitoring.
\end{abstract}

\section{INTRODUCTION}

The geometric calibration techniques for space remote sensing sensors are usually divided into laboratory calibration and onorbit calibration. To be specific, in (Liu, Jia and Ding, 2010) and (Wu, Han and $\mathrm{He}, 2007)$, laboratory calibration is introduced to calibrate geometric parameters deviating from the rated value. Such deviation is resulted from errors in camera equipment processing and installation before launching. However, after the satellite's launch and during its operation, the laboratory calibration value is no longer applicable and the geometric parameters can also induce unpredictable changes, because of the changes in pressure, vibration, temperature, and other aspects. To deal with the above issue, on-orbit calibration technique is needed, as discussed in (Wang et al., 2014), (Zhang et al., 2014), and (Li and Wang, 2012). Currently, the method based on a geometric calibration site is usually adopted for onorbit calibration, as described by (Breton et al., 2002), (Radhadevi et al., 2011), (Zhang, 2012), and (Wang et al., 2017). However, this method is significantly affected by weather, topography and other conditions. Furthermore, due to the long update cycle and poor timeliness, it cannot be applied to perform timely camera geometric parameters monitoring. (Delevit et al., 2012).

In this paper, we propose a real-time calibration method that applies the optical auto-collimation principle to on-orbit monitoring and realizes a solution for the key geometric parameters. By installing collimated light sources, area-array CCDs, prisms, and other devices inside the satellite load system, changes in the camera parameters are converted into changes in the spot images. By extracting and processing the spot images, we can solve the variation of the camera parameters to achieve fast and efficient on-orbit monitoring.

\section{THE PRINCIPLE OF ONBOARD MONITORING}

\subsection{Optical Auto-collimation Principle}

The optical auto-collimation principle is an optical phenomenon that the object and the image lie in the same or conjugate planes. Specifically, when a light source is used to irradiate the reticule on the focal plane of the objective lens, the point $\mathrm{O}$ enters the objective lens and returns to its original paths after being reflected by the plane mirror, finally being imaged at the position of source point $\mathrm{O}$. At this time, if the incident light is kept unchanged and the plane mirror is rotated by angle $\theta$, the emergent light is rotated by an angle of $2 \theta$ and finally imaged at point $\mathrm{O}^{\prime}($ Zhang, Fan and Cao, 2006), as shown in Figure 1.

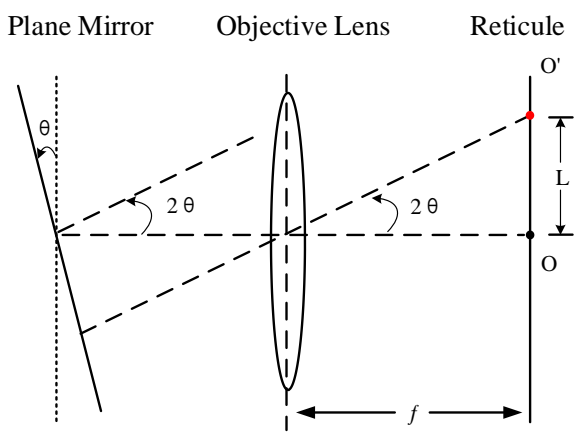

Figure 1. Optical auto-collimation schematic

Denote the distance between the emission point and the imaged point by $\mathrm{L}$, with a focal length $f$. 
Then:

$$
L=f \cdot \tan 2 \theta
$$

The figure shows the situation where the position of the reticule does not move, while the plane mirror is rotated at a certain angle. This principle is equally applicable when the mirror remains unchanged and the reticule moves, such as reticule rotation around the coordinate axis, parallel to forward or backward, and so on.

\subsection{Design of Onboard Monitoring Device}

In this paper, a dual-line-array camera is utilized as an example to design a geometric parameter onboard monitoring system.

The analysis and design procedure is as follows. According to subsection 2.1, the system first needs a device that emits light and records the spot image. To achieve this goal, the easiest way is taking the camera's lens as the objective lens and focal plane as the imaging plane. Because it is a linear-array CCD, we consider the installation at both ends, and the collimated light source is installed next to the spot imaging device. A laser emission device is used to emit a collimated light source, and the spot recording device is used to record the changes in the spot positions. Then, to make the emitted light reflect along the original light path, a reflecting prism is installed. The reflecting prism is placed on a photographic base and located at the center of the camera system, and thus it can be considered as a camera system benchmark. In addition, for the purpose of guiding light and preventing light scattering, a collimator tube is installed.

When the laser diode of the emission device emits light, the light beam reaches the main mirror of the camera after being reflected by the system. Then, it passes through the reflection and conduction of prisms and collimator tubes to reach the reflector of the central prism. The beam is again reflected by the central prism and returns according to the original paths, and eventually being imaged on the spot recording area-array CCD.

Taking the light beam emitting at one end as an example, a simplified device schematic can be shown in Figure 2. The solid line represents the light beam emitted from the area-array $\mathrm{CCD}$, and the dotted line represents the light beam propagating in the reverse direction after reaching the central prism. Figure 3 shows the linear-array CCD and transceivers in the focal plane.

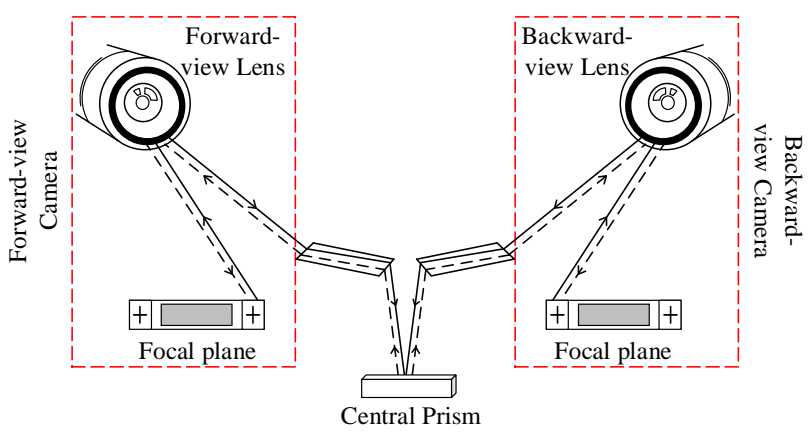

Figure 2. Device simplified diagram

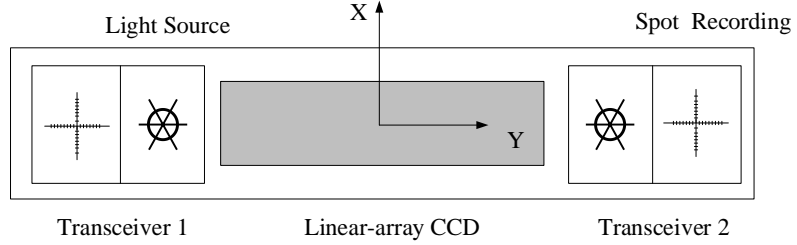

Figure 3. Schematic diagram of focal plane

\subsection{Data Processing Flow}

The spot image is obtained and the center point coordinates are extracted. Through comparison with the rated center point coordinates, the changes in the focal length and the rotation angles of visual axis around the $\mathrm{X}, \mathrm{Y}, \mathrm{Z}$-axis are obtained. Selecting the Forward-view camera as a datum, the data processing diagram is as follows:

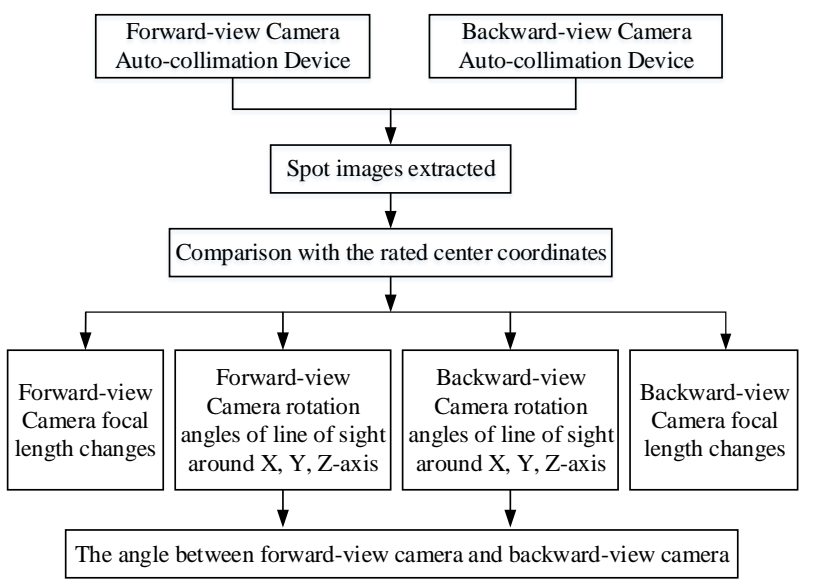

Figure 4. Data processing flow chart

\section{PRINCIPLE ANALYSIS}

We herein take the forward-view camera as an example to simulate the spatial relationship between the camera lens and the linear-array CCD. Assuming that the camera lens does not move, and the position of the linear-array CCD changes, Figure 5 shows the initial positional relationship between them.

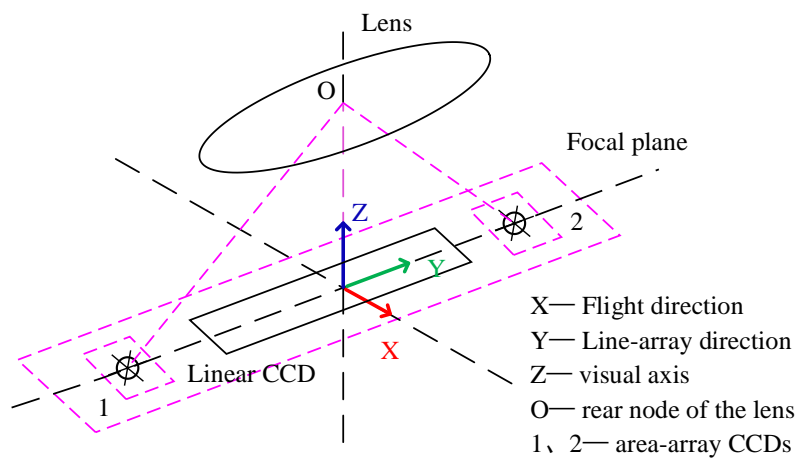

Figure 5. Initial positional relationship

\subsection{Change in focal length}

As shown in Figure 6, when the linear-array CCD is translated upward, the visual axis is fixed, while the focal length changes. 


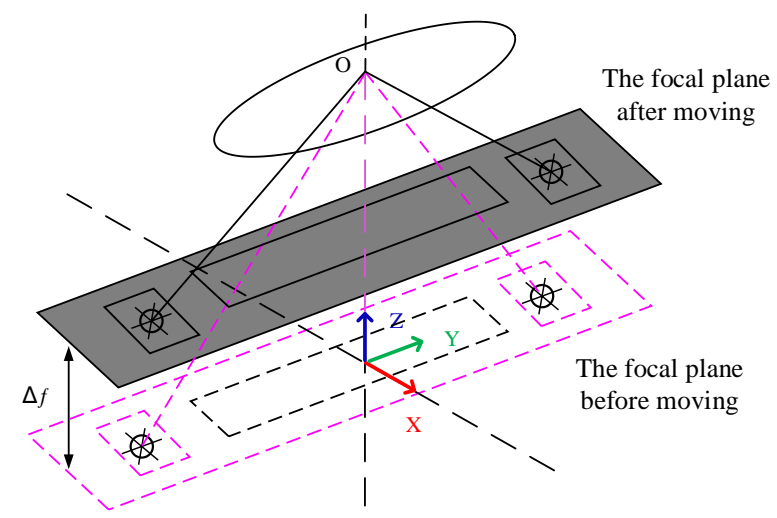

Figure 6. Change in focal length

Figure 7 shows the positions of the spots before and after the movement.

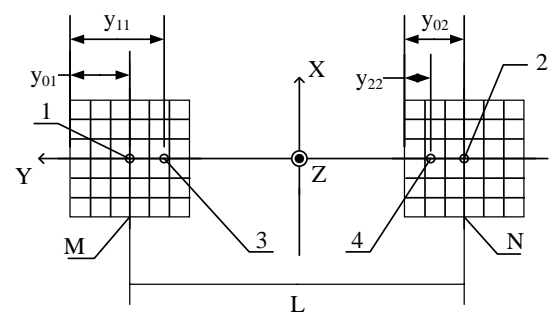

M、 N-Spot imaging area-arrays

1、2-Rated imaging positions

3、4-Actual imaging positions

$\mathrm{L}$ - The distance between the two area-array centers

Figure 7. Changes in spot positions

The change in focal length affects the y-coordinate of the spot. $\Delta y$ can be determined by the average of the difference in the positions of the imaging points between the two cases:

$$
\Delta y=\frac{\left(y_{11}-y_{01}\right)\left(y_{02}-y_{22}\right)}{2} \delta
$$

where $\quad \delta=$ pixel size

$f=$ effective focal length

According to Figure 7, we can obtain the variation of focal length $\Delta f$ as:

$$
\Delta f=\left[\frac{\left(y_{11}-y_{01}\right)+\left(y_{02}-y_{22}\right)}{2} \delta\right] \cdot \frac{f}{L}
$$

\subsection{Visual axis rotates around the $\mathrm{X}$-axis}

When the linear-array CCD moves along the Y-axis, a specific angle is formed between the visual axis and the optical axis, which is equivalent to the case where the visual axis is rotated by a certain angle $\Delta \beta$ around the $\mathrm{X}$-axis. Figure 8 is a simulation diagram, and Figure 9 shows the positions of the spots.

To obtain the rotation angle $\Delta \beta$ :

$$
\Delta \beta=\arctan \left[\left[\frac{\left(y_{01}-y_{11}\right)+\left(y_{02}-y_{22}\right)}{2} \delta\right] \cdot \frac{\cos ^{2} \beta}{2 f}\right]
$$

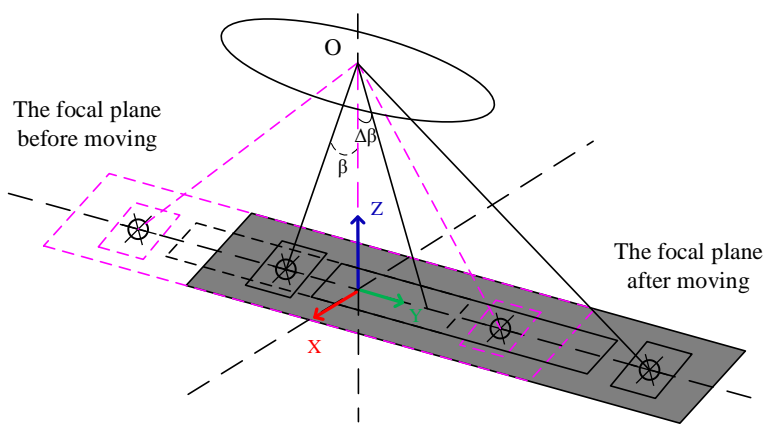

Figure 8 . Visual axis rotates around the $\mathrm{X}$-axis

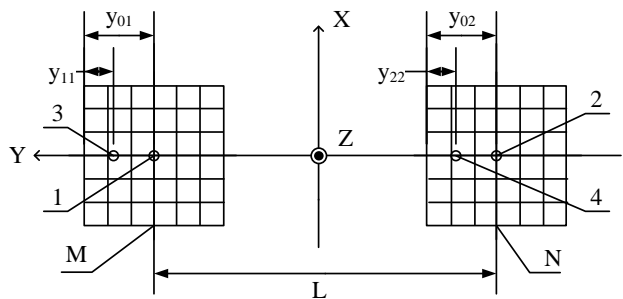

M、 N-Spot imaging area-arrays

1、2-Rated imaging positions

3、4-Actual imaging positions

$\mathrm{L}$ - The distance between the two area-array centers

Figure 9. Changes in spot positions

When the value of $\mathrm{x}$ is very small, the value of $\tan (\mathrm{x})$ or $\arctan (\mathrm{x})$ can be approximated as $\mathrm{x}$. Then, the formula (4) can be simplified as follows:

$$
\Delta \beta=\left[\frac{\left(y_{01}-y_{11}\right)+\left(y_{02}-y_{22}\right)}{2} \delta\right] \cdot \frac{\cos ^{2} \beta}{2 f}
$$

\subsection{Visual axis rotates around the $\mathrm{Y}$-axis}

When the linear-array CCD moves along the $\mathrm{X}$-axis, a specific angle is formed between the visual axis and the optical axis, which is equivalent to the case where the visual axis is rotated by a certain angle $\Delta \alpha$ around the Y-axis. Figure 10 is a simulation diagram, and Figure 11 shows the positions of the spots.

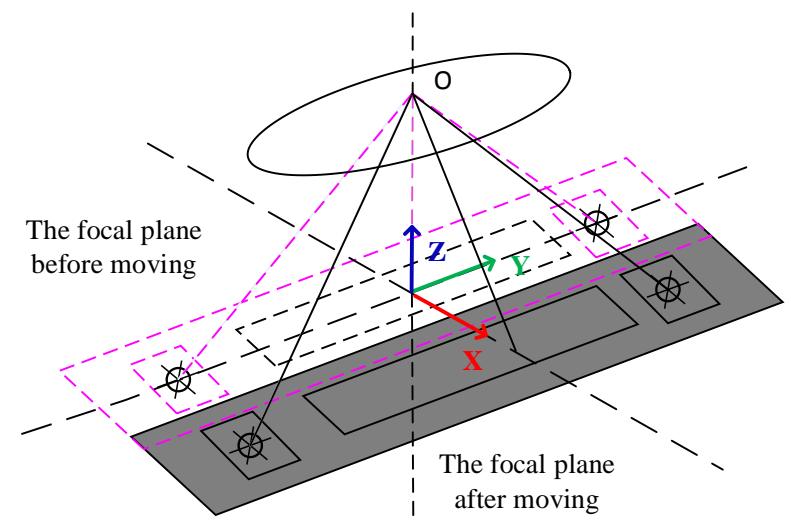

Figure 10. Visual axis rotates around the $\mathrm{X}$-axis 


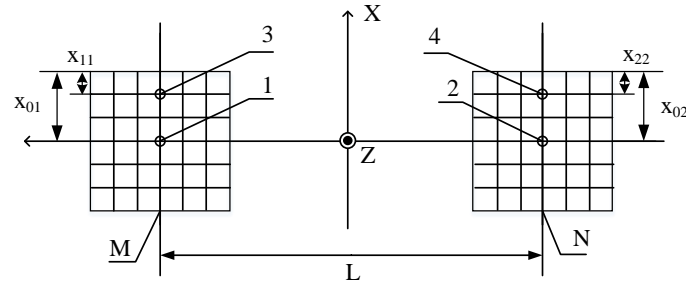

M、 N-Spot imaging area-arrays

1、2-Rated imaging positions

3、4-Actual imaging positions

L-The distance between the two area-array centers

Figure 11. Changes in spot positions

Similar to Section 3.2, the rotation angle $\Delta \alpha$ :

$$
\Delta \alpha=\left[\frac{\left(x_{01}-x_{11}\right)+\left(x_{02}-x_{22}\right)}{2} \delta\right] \cdot \frac{\cos ^{2} \alpha}{2 f}
$$

\subsection{The camera rotates around the visual axis}

As shown in Figure 12, the linear-array CCD rotating about the visual axis is equivalent to the camera rotating about the viewing axis. Figure 13 shows the positions of the spots.

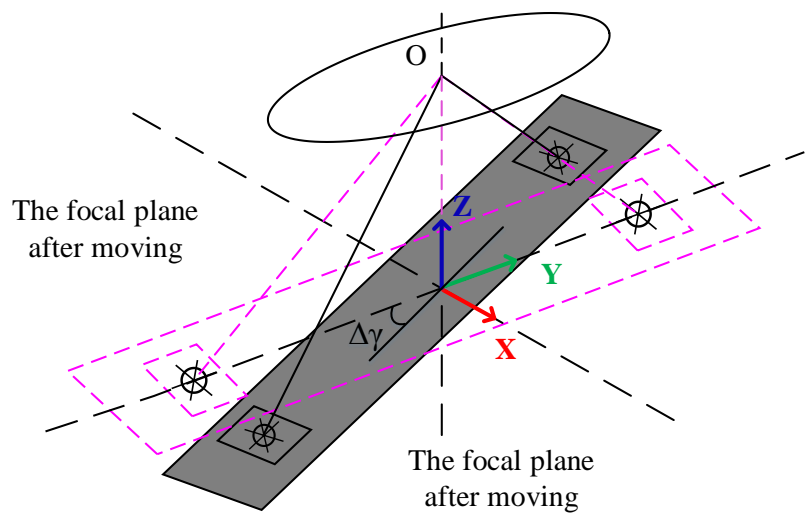

Figure 12. The camera rotates around the visual axis

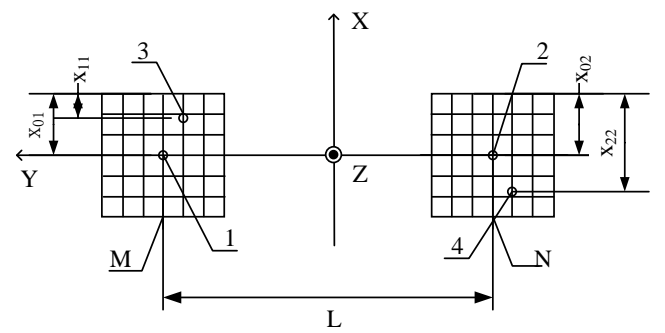

M、 N-Spot imaging area-arrays

1、2-Rated imaging positions

3、4-Actual imaging positions

L-The distance between the two area-array centers

Figure 13. Changes in spot positions

Both the X-coordinate and Y-coordinate of the spot image are changed, but the Y-coordinate changes little. Thus, it can be ignored. Then the rotation angle $\Delta \gamma$ is:

$$
\Delta \gamma=\left[\frac{\left(x_{01}-x_{11}\right)+\left(x_{22}-x_{02}\right)}{L}\right] \cdot \delta
$$

\subsection{Formula summary}

The preceding is derived in the case of only one variable change, but the situation is much more complex when the satellite is actually on-orbit and it is not clear which parameters have changed. It is known that $\Delta f$ and $\Delta \beta$ cause a change in the Ycoordinate of the spot image, and $\Delta \alpha$ and $\Delta \gamma$ cause a change in the $\mathrm{X}$-coordinate. Therefore, we can obtain the expressions when the four parameters change indefinitely according to the spot image coordinate.

$$
\begin{gathered}
\Delta f=\left(\frac{Y_{L}-Y_{R}}{4}\right) \cdot \frac{\delta f}{L} \\
\Delta \beta=\left(\frac{Y_{L}+Y_{R}}{4}\right) \cdot \frac{\delta \cos ^{2} \beta}{f} \\
\Delta \alpha=\left(\frac{X_{L}+X_{R}}{4}\right) \cdot \frac{\delta \cos ^{2} \alpha}{f} \\
\Delta \gamma=\left(\frac{X_{L}-X_{R}}{L}\right) \cdot \delta
\end{gathered}
$$

where $\quad Y_{R}, Y_{L}=\mathrm{Y}$-changes of right and left area-arrays $X_{R}, X_{L}=\mathrm{X}$-changes of right and left area-arrays

\section{EXPRIMENT AND DISCUSSION}

\subsection{Experiment principle and feasibility verification}

Two optical autocollimators, two plane mirrors, a threedimensional turntable, and two theodolites are used to build an experimental platform. Each group of auto-collimation devices contains an autocollimator and a plane mirror, measuring the rotation angle of the visual axis around the $\mathrm{X}, \mathrm{Y}, \mathrm{Z}$-axis, processing and analysing the data obtained to preliminarily verify the feasibility of applying the auto-collimation principle to on-orbit monitoring.

In the laboratory, because the autocollimator lens and the focal plane are solidly connected together, the relative position cannot be changed. Therefore, the rotation of the camera lens around the three axes is simulated by rotating the plane mirrors, and the experiment does not simulate the situation with a flexible focal length.

The experimental platform is shown in Figure 14. Autocollimation device 1 simulates the linear-array CCD imaging process, and auto-collimation device 2 simulates the imaging process of an area-array CCD at one end. Two autocollimators are placed with a specific angle relative to each other, and the optical axis of the autocollimators coincide with the central axis of the plane mirrors. Plane mirrors are mounted on a threedimensional turntable and can be rotated by $360^{\circ}$. For autocollimation device 1 , the horizontal axis is set as the $\mathrm{Y}$-axis, the vertical axis is the $\mathrm{X}$-axis, and the $\mathrm{Z}$-axis is the rotation axis of the turntable; this system is denoted by coordinate system 1$\mathrm{XYZ}$. For auto-collimation device 2, its coordinate system is denoted 2-XYZ, where the $\mathrm{X}$-axis and $\mathrm{Y}$-axis have a specific angle between them that is the same as that of 1-XYZ. Figure 15 is the coordinate system diagram. 


\begin{tabular}{|c|c|c|c|c|c|c|c|}
\hline \multirow{2}{*}{\multicolumn{2}{|c|}{$\begin{array}{l}\text { Rotation around } \\
\text { the axis (") }\end{array}$}} & \multicolumn{2}{|c|}{$\mathrm{X}$-axis } & \multicolumn{2}{|c|}{ Y-axis } & \multicolumn{2}{|c|}{ Z-axis } \\
\hline & & \multirow{2}{*}{$\begin{array}{c}\text { Mirror } 1 \\
0.040000\end{array}$} & \multirow{2}{*}{$\begin{array}{l}\text { Mirror } 2 \\
0.030000 \\
\end{array}$} & \multirow{2}{*}{$\begin{array}{c}\text { Mirror } 1 \\
0.150000 \\
\end{array}$} & \multirow{2}{*}{$\begin{array}{c}2 / \sqrt{3} \cdot \text { Mirror } 2 \\
0.046188\end{array}$} & \multirow{2}{*}{$\begin{array}{c}\text { Mirror } 1 \\
0.040000 \\
\end{array}$} & \multirow{2}{*}{$\frac{2 / \sqrt{3} \cdot \text { Mirror } 2}{0.046188}$} \\
\hline \multirow{11}{*}{$\begin{array}{l}\text { Turntable } \\
\text { rotation } \\
\text { angle (") }\end{array}$} & 0 & & & & & & \\
\hline & 72 & 70.980000 & 71.160000 & 72.000000 & 71.822373 & 62.310000 & 71.949391 \\
\hline & 144 & 143.170000 & 143.390000 & 144.220000 & 144.002704 & 124.630000 & 143.910328 \\
\hline & 216 & 215.270000 & 215.640000 & 215.540000 & 216.183035 & 187.000000 & 215.929001 \\
\hline & 288 & 286.920000 & 287.290000 & 288.330000 & 288.536571 & 249.060000 & 287.589716 \\
\hline & 360 & 358.720000 & 359.140000 & 360.410000 & 360.786183 & 311.890000 & 360.139551 \\
\hline & 432 & 431.050000 & 431.700000 & 432.540000 & 432.966514 & 374.040000 & 431.904189 \\
\hline & 504 & 503.280000 & 503.930000 & 504.510000 & 505.031374 & 436.310000 & 503.807392 \\
\hline & 576 & 575.180000 & 575.890000 & 577.130000 & 577.304081 & 498.610000 & 575.745235 \\
\hline & 648 & 647.180000 & 647.940000 & 648.860000 & 649.288113 & 560.970000 & 647.752361 \\
\hline & 720 & 718.990000 & 719.720000 & 721.080000 & 721.549272 & 623.230000 & 719.644017 \\
\hline \multicolumn{2}{|c|}{$\begin{array}{l}\text { Mean difference } \\
\text { (") }\end{array}$} & \multicolumn{2}{|c|}{0.460909} & \multicolumn{2}{|c|}{0.340353} & \multicolumn{2}{|c|}{0.177646} \\
\hline
\end{tabular}

Table 1. Experimental data

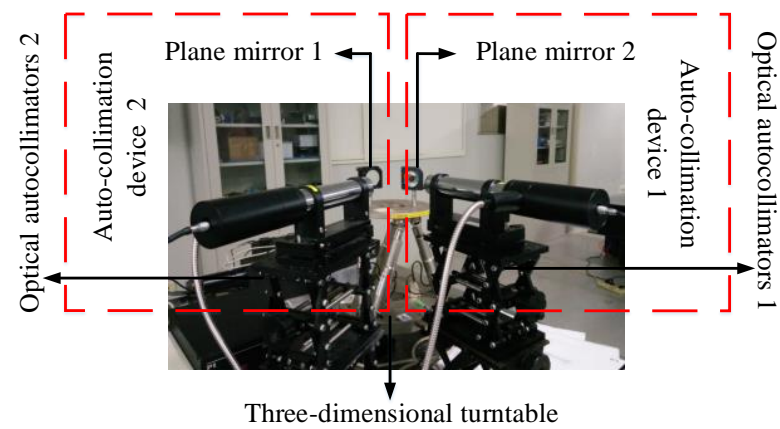

Figure 14. Experimental platform

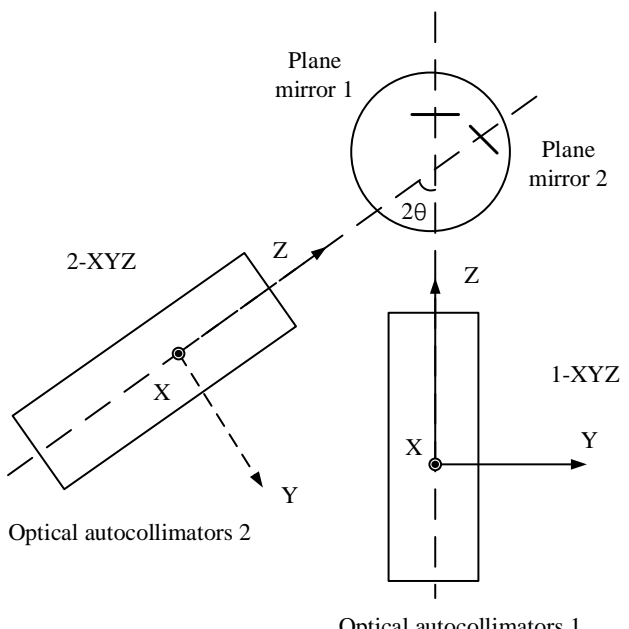

Figure 15. Coordinate system diagram

The rotation angle of auto-collimation device 1 around the coordinate $1-\mathrm{XYZ}$ is the angle that the visual axis rotates around the image-space coordinate system. When the turntable rotates by a certain angle, plane mirror 1 rotates by the same angle. Plane mirror 2 rotates by a different angle around its own coordinate 2-XYZ, but there should be a certain numerical relationship between the two rotation angles. Therefore, the purpose of the experiment is to verify whether the two plane mirrors rotate their corresponding angles around their respective coordinate axis system, when the turntable rotates a certain angle. If satisfied, the feasibility of using the auto-collimation principle for on-orbit monitoring can be verified.

\subsection{Experimental results and discussion}

When the turntable rotates around the $\mathrm{X}$-axis of $1-\mathrm{XYZ}$ by angle $\Delta \beta$, the rotation angles of plane mirrors 1 and 2 are both $\Delta \beta$. When the turntable rotates by $\Delta \alpha$ around the Y-axis of 1$\mathrm{XYZ}$, plane mirror 1 rotates by $\Delta \alpha$ while the rotation angle of plane mirror 2 about the $\mathrm{Y}$-axis of its own coordinate system 2$\mathrm{XYZ}$ is $\Delta \alpha \cdot \cos \beta$. When the turntable rotates by $\Delta \gamma$ around the $\mathrm{Z}$-axis of $1-\mathrm{XYZ}$, plane mirror 1 rotates by $\Delta \gamma$ and the rotation angle of plane mirror 2 about the $Z$-axis of its own coordinate system $2-\mathrm{XYZ}$ is $\Delta \gamma \cdot \sin \beta$.

We have conducted numerous tests with steps of $0.01^{\circ}, 0.02^{\circ}$, $0.03^{\circ}$, with $\theta$ values of $15^{\circ}$ and $30^{\circ}$. Table 2 lists the results for the condition in which the step is $0.02^{\circ}\left(72^{\prime \prime}\right)$, the angle of plane mirrors is $30^{\circ}$ when rotated about the $\mathrm{X}$-axes and $\mathrm{Y}$-axes, and the angle of plane mirrors is $60^{\circ}$ when rotated about the $\mathrm{Z}$-axis.

The experimental data show that the rotating angle of plane mirrors 1 and 2 around the three axes agrees with the theoretical value during the rotation of the turntable from 0 " to 720," and the average error does not exceed 0.5." This proves that when the camera's visual axis rotates around three axes, it can be measured by the auto-collimation devices at both ends of the linear-array CCD. It is feasible to use the auto-collimation principle for on-board monitoring of the geometric parameters.

\subsection{Accuracy analysis}

The accuracy of the optical autocollimator is an important factor affecting the monitoring accuracy. The ELCOMAT HR produced in Germany is the most accurate photoelectric autocollimator on the market, which has a display resolution of 0.005 " and a measurement range of \pm 150 ." Its indication error is \pm 0.01 " over a $10 "$ range, and its measurement uncertainty is 
$\pm 0.03 "$ over the full range. Experiments are performed using optical autocollimators with an accuracy of \pm 1 ." The average accuracies of $\Delta \beta, \Delta \alpha$, and $\Delta \gamma$ obtained by our experiments are $0.55, " 0.64, "$ and $0.97, "$ respectively, both less than $1 . "$

Although the error of $\Delta \gamma$ is larger, it is a rotation angle in the image plane and can be better eliminated by the forward overlap during photogrammetric processing. If more accurate autocollimation equipment is available, higher monitoring accuracy can be achieved. When machining the auto-collimation device, the placement accuracy around the $\mathrm{X}$ and $\mathrm{Y}$-axes can also be improved, leaving the error that cannot be eliminated around the $\mathrm{Z}$-axis, thus improving the monitoring accuracy.

\section{CONCLUSION}

To achieve real-time on-orbit calibration, in this paper we have proposed an onboard monitoring technology for space optical camera based on the optical auto-collimation principle. The proposed method employs a set of optical auto-collimation devices for optical camera, and solves the variation of key geometric parameters by real-time spot image processing. We have analysed the principle of this monitoring technology, and set up an experimental platform to simulate the imaging relationship of the device. In addition, the monitoring accuracy is analysed. The result verifies the feasibility of this method, which provides a novel research idea for real-time on-orbit calibration.

\section{REFERENCES}

Breton, E., Bouillon, A., Gachet, R., \& Delussy, F., 2002. Preflight and in-flight geometric calibration of SPOT5 HRG and HRS images. INTERNATIONAL ARCHIVES OF PHOTOGRAMMETRY REMOTE SENSING AND SPATIAL INFORMATION SCIENCES, 34(1), 20-25.

Delvit, J. M., Greslou, D., Amberg, V., Dechoz, C., de Lussy, F., Lebegue, L., ... \& Bernard, L., 2012. Attitude assessment using Pleiades-HR capabilities. Proceedings of the International Archives of the Photogrammetry, Remote Sensing and Spatial Information Sciences, Melbourne, VIC, Australia, 25.

Li, D. R., \& Wang, M., 2012. On-orbit geometric calibration and accuracy assessment of ZY-3. Spacecr. Recover. Remote Sens, 33, 1-6.

LIU, B., JIA, J. Q., \& DING, Y. L., 2010. Geometric calibration with angle measure for $\mathrm{CCD}$ aerial photogrammetric camera in laboratory [J]. Laser \& Infrared, 3, 019.

Radhadevi, P. V., Müller, R., d'Angelo, P., \& Reinartz, P., 2011. In-flight geometric calibration and orientation of ALOS/PRISM imagery with a generic sensor model. Photogrammetric Engineering \& Remote Sensing, 77(5), 531-538.

Wang, J., Wang, R., Hu, X., \& Su, Z., 2017. The on-orbit calibration of geometric parameters of the Tian-Hui 1 (TH-1) satellite. ISPRS Journal of Photogrammetry and Remote Sensing, 124, 144-151.

Wang, M., Yang, B., Hu, F., \& Zang, X., 2014. On-orbit geometric calibration model and its applications for highresolution optical satellite imagery. Remote Sensing, 6(5), 43914408 .
WU, G. D., HAN, B., \& HE, X., 2007. Calibration of geometric parameters of line-array CCD camera based on exact measuring angle in lab [J]. Optics and Precision Engineering, 10, 029.

Yongsheng, Z., 2012. Design and implementation of Songshan test field for high resolution remote sensing and mapping. Journal of Geomatics Science and Technology, 2, 000.

ZHANG, J. Y., FAN, T. Q., \& CAO, X. D., 2006. Autocollimation angular measurement method based on imaging grating. Opto-Electronic Engineering, 11, 012.

Zhang, Y., Zheng, M., Xiong, J., Lu, Y., \& Xiong, X., 2014. On-orbit geometric calibration of ZY-3 three-line array imagery with multistrip data sets. IEEE Transactions on Geoscience and Remote Sensing, 52(1), 224-234. 